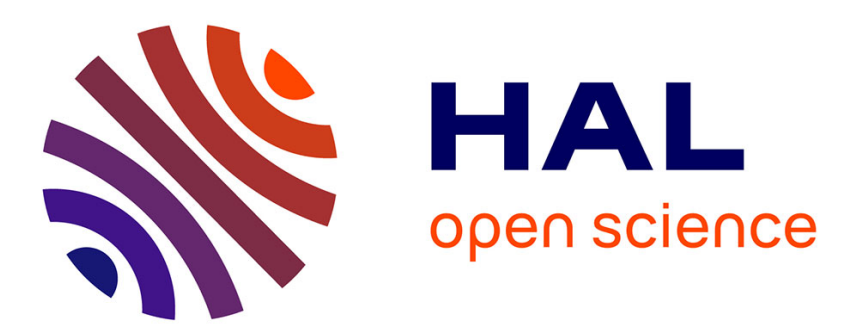

\title{
Microfluidic compartmentalization of diffusively coupled oscillators in multisomes induces a novel synchronization scenario
}

Marcello A Budroni, Kristian Torbensen, Ottorino L Pantani, Sandra Ristori, Federico Rossi, Ali Abou-Hassan

\section{To cite this version:}

Marcello A Budroni, Kristian Torbensen, Ottorino L Pantani, Sandra Ristori, Federico Rossi, et al.. Microfluidic compartmentalization of diffusively coupled oscillators in multisomes induces a novel synchronization scenario. Chemical Communications, 2020, 56, pp.11771-11774. 10.1039/D0CC05046F . hal-03020840

\section{HAL Id: hal-03020840 \\ https: / hal.sorbonne-universite.fr/hal-03020840}

Submitted on 24 Nov 2020

HAL is a multi-disciplinary open access archive for the deposit and dissemination of scientific research documents, whether they are published or not. The documents may come from teaching and research institutions in France or abroad, or from public or private research centers.
L'archive ouverte pluridisciplinaire HAL, est destinée au dépôt et à la diffusion de documents scientifiques de niveau recherche, publiés ou non, émanant des établissements d'enseignement et de recherche français ou étrangers, des laboratoires publics ou privés. 


\section{Microfluidic compartmentalization of diffusively coupled oscillators in multisomes induces a novel synchronization scenario}

Received 00th January 20xx, Accepted 00th January 20xx DOI: $10.1039 / \times 0 \times x 00000 x$

\author{
Marcello A. Budroni, ${ }^{a+}$ Kristian Torbensen, ${ }^{\mathrm{b}+}$ Ottorino L. Pantani, ${ }^{\mathrm{c}}$ Sandra Ristori, ${ }^{\mathrm{d}}$ Federico Rossi, ${ }^{* e}$ \\ and Ali Abou-Hassan*b
}

Stable cell like multisomes encapsulating the inorganic chemical oscillator Belousov-Zhabotinsky were engineered and organized in a linear network of diffusively-coupled chemical oscillators by using microlfuidics. The multi-compartmentalization and the spatial configuration resulted in a new global synchronization scenario. After an initial induction interval, all the oscillators started to pulsate in phase with a halved period respect to the natural one.

The imitation of biological life-like functions in synthetic engineered systems is one of the main goals of emerging disciplines like Systems Chemistry and Synthetic Biology. ${ }^{1}$ Among others, here we are interested in processes that can give birth to cooperative effects and synchronization which are ubiquitous in nature and manifest themselves as coordinated oscillations in one or more characteristic parameters (heartbeat, circadian clocks, calcium signalling, etc). ${ }^{2}$ In biological systems, cooperation and synchronization occurs via chemical communication based on a messenger diffusion/reaction, which extends over a wide range of time and length-scales. ${ }^{3}$ In this context, interfaces and membranes play a fundamental role to control the communication and the emergent global dynamics, including synchronization. Therefore, building a compartmentalised oscillating model where liquid/liquid interfaces can be tuned and engineered

\footnotetext{
a. Department of Chemistry and Pharmacy, University of Sassari, Via Vienna 2, 07100 Sassari, Italy.

b. Sorbonne Université, CNRS UMR 8234, PHysico-chimie des Electrolytes et Nanosystèmes InterfaciauX (PHENIX), F- 75005 Paris, France.

Ali.abou hassan@sorbonne-universite.fr

Department of Agrifood Production and Environmental Sciences, University of Florence P.le delle Cascine 28, 50144 Firenze, Italy

d. Department of Chemistry \& CSGI, University of Florence, 50019 Sesto Fiorentino, Firenze, Italy

e. Department of Earth, Environmental and Physical Sciences - DEEP Sciences University of Siena, Pian dei Mantellini 44, 53100 Siena, Italy. Federico.rossi2@unisi.it

+ Equal contribution.

Electronic Supplementary Information (ESI) available: [microfluidic generation of droplets, mathematical model, bromine phase transfer experiments and dynamic light scattering experiments]. See DOI: 10.1039/x0xx00000x
}

precisely, can help scientists to understand the communication mechanisms and the synchronicity observed in nature.

The Belousov-Zhabotinsky (BZ) reaction, is a well-known robust chemical oscillator which has been used as a model system for bio-inspired chemical communications studies, when encapsulated inside coupled microcompartments. ${ }^{4}$ Assisted by droplet microfluidics, BZ cell-like oscillators with precise dimensions and defined interfacial properties have been elaborated. They include oscillating droplets, ${ }^{5}$ emulsions, ${ }^{6}$ liposomes $^{7}$ and polymersomes. ${ }^{8}$ The coupling among BZ droplets was modulated either by changing the chemistry of the continuous phase and the droplets spatial organization, ${ }^{9}$ or by manipulating at the molecular level the reactivity ${ }^{10}$ and the permeability ${ }^{11}$ of the messenger species at the interface. The membrane lamellarity was also modulated at the molecular level allowing to tune the chemical communication. ${ }^{11 b, 12}$ In this case a precise control of the interfacial structure and chemical composition was reached by using advanced small angle scattering techniques, such as high resolution synchrotron SAXS. ${ }^{13}$

However, in all these examples the resulting dynamical patterns were always found to be dominated by an inhibitory character, with few exceptions. ${ }^{10 a}$, 14 Motivated by previous experiments in a 2-dimensional arrangement, where pairs of phospholipidstabilised BZ droplets could oscillate synchronously for a short time, ${ }^{15}$ here we explore a 1-dimensional array of multisomes with the aim of obtaining an activatory-modulated coupling in a configuration never explored before.

Bio-synthetic multisomes are water/oil/water (w/o/w) double emulsions (DEs) stabilized by amphiphilic lipids. ${ }^{16}$ They are used as a model for cell-like compartments but also as precursors for templating vesicles using microfluidics. ${ }^{17}$ In a multi-scale bioinspired methodology, DEs offer the possibility to investigate the effect of multi-compartmentalization and multi-interfaces in cells where partition occurs in different pseudophases composing the system. Such effects can have a direct impact on chemical communication and give birth to new global dynamics. Herein, we successfully obtained for the first time stable BZ 
oscillating multisome droplets stabilized by a layer of lipid amphiphile (1,2-Dimyristoyl-sn-glycero-3-phosphocholine, DMPC). The choice of this lipid was motivated by its property of forming fluid bilayers at room temperature, while not presenting unsaturations which could interfere with the BZ reactants and intermediates. Moreover, assisted by microfluidics the compartments were organized into a 1-D array and the collective dynamics of the system was studied. The resulting macroscopic behaviour was a synchronization inphase to a single dominant period, different from the natural period of the uncoupled oscillators.

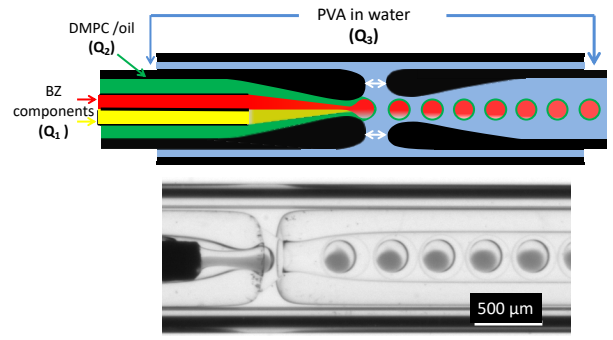

Scheme 1. Top) Schematic illustration of the microfluidic device used for generating w/o/w double emulsions (see details in ESI). Bottom) Optical microscopy image showing the real-time the formation of BZ double emulsions. (see video 1, ESI)

Encapsulating $\mathrm{BZ}$ in DEs is challenging due to the acidic $\mathrm{pH}$ and the oxidizing potential of ferroin and bromate as well as the different species generated in the BZ reaction. Moreover, such species are active at the interfaces and, in addition to the evaporation of the organic oil shell, they can contribute to destabilize the final DEs. To start, BZ DEs were generated using a home-built glass microfluidic device based on co-flow and flow focusing geometries as shown in scheme 1 (see ESI for more details). ${ }^{18}$ In order to avoid oscillations to occur prior to encapsulation, the microfluidic setup was configured to allow $\mathrm{BZ}$ reagents to be mixed in situ while monitored by optical microscopy. As we aim to study the effect of multicompartmentalization and interfaces in a DEs configuration, the organic shell should be preserved. Consequently, to avoid its evaporation while allowing spatial 1D organization, the DEs were collected in a glass capillary tube which was sealed in both ends with wax before optical monitoring (see the Top Panel of Figure 1 and ESI for more information). To infer the asymptotic behaviour of the network, communicating droplets have to be physically stable for a long time (at least 30 minutes). Organizing the DEs in the capillary tube prevented the oil shell from evaporation, however popping up of the inner BZ core was observed depending on the formulation conditions. When the external phase was of the same composition as the internal phase ( $2 \% \mathrm{w} / \mathrm{w}$ PVA) some of the DEs were observed to swell followed by rupturing of their internal BZ/oil interface. This interface instability may be provoked by the difference in the osmotic pressure across the oil shell related the presence of the BZ-components in the inner core. Such a difference can induce diffusion of water from the outside towards the inside of the DE and weaken the lipid assembly at the interface.
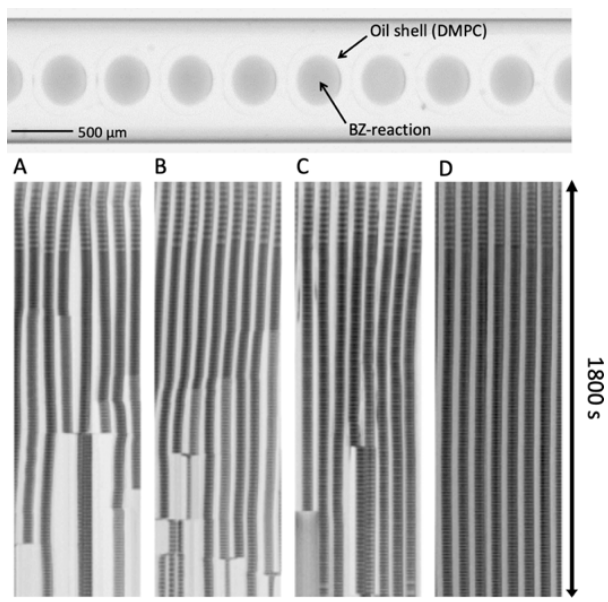

Figure 1. Top) DEs encapsulating BZ and arranged in a 1D array. Bottom) Space-time plots obtained by taking vertical projections of the recorded image sequences of double emulsion 1D arrays (sampling time $1 \mathrm{~s}$ ). Bright lines correspond to oxidation oscillations, while dark regions represent the oscillator in the reduced state. The BZ composition was fixed as described in ESI. The outer suspending phase was (A) 2\% w/w PVA, (B) $2 \% \mathrm{w} / \mathrm{w}$ PVA and 1.445M sucrose, (C) 5\% w/w PVA and 1.445M sucrose and (D) 10\% w/w PVA and $1.445 \mathrm{M}$ sucrose (see video $2, \mathrm{ESI}$ )

To check this hypothesis, sucrose was added at a concentration of $1.445 \mathrm{M}$ to the outer phase to equilibrate the stress in DEs (see ESI). We found that the life-time of the double emulsions was thus prolonged to several hours, indicating that sucrose exerts a favourable effect on the osmotic balance between the inner and outer phases. Typical experimental dynamics without sucrose in the outer phase is shown in Figure 1A. Here the disappearance of many oscillating DEs takes place after a few hundred seconds. The addition of sucrose improved the DEs stability (Figure 1B). However, a further common feature observed in Figure $1 A$ and $B$ is the horizontal shift in position of the individual space-time plots. This is due to DEs movement over time in the tube, exerting some mechanical stress that contributes to the overall instability. Such mechanical stress could be caused by pressure waves arising in the system upon the rupturing of nearby double emulsion droplets. To circumvent this problem, the viscosity of the outer phase was increased by using higher concentrations of the viscosity modulator polyvinyl alcohol (PVA). Figures $1 \mathrm{C}$ and $1 \mathrm{D}$ show the effect of adding 5 and $10 \% \mathrm{w} / \mathrm{w}$ (PVA) to the sucrose containing outer phase, respectively. A clear improvement on the overall stability and fixation of the double emulsions was observed in the case of $10 \% \mathrm{w} / \mathrm{w}$ PVA (Figure 1D).

As an example of the global dynamics of the whole array, Figure $2 \mathrm{~A}$ shows the behaviour of 4 coupled microoscillators extracted from experimental timeseries in Figure 1D. All the microoscillators in the glass capillary progressively synchronize in-phase to a single dominant period (Figure 2B-C). In fact, when the inhibitor is sequestrated from the core of the microoscillators there is a short initial oxidized phase ${ }^{19}$ and, after about 15 oscillatory cycles, the period halves from an averaged value $\tau^{\prime} \sim 35 \mathrm{~s}$ to a value $\tau^{\prime \prime} \sim 18 \mathrm{~s}$. At the same time the phase difference between adjacent and alternate oscillators 


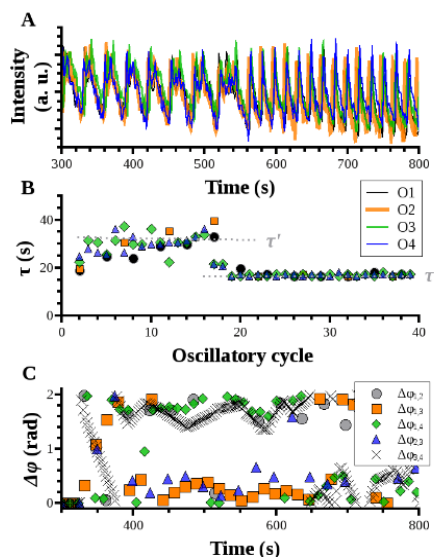

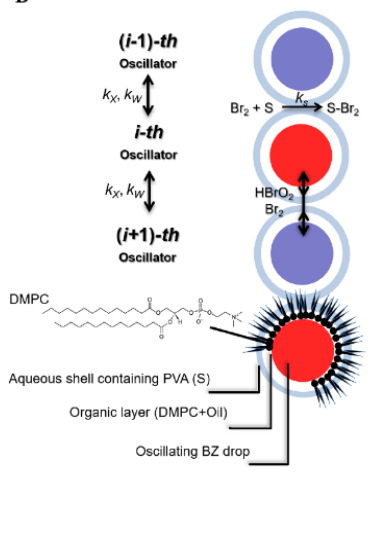

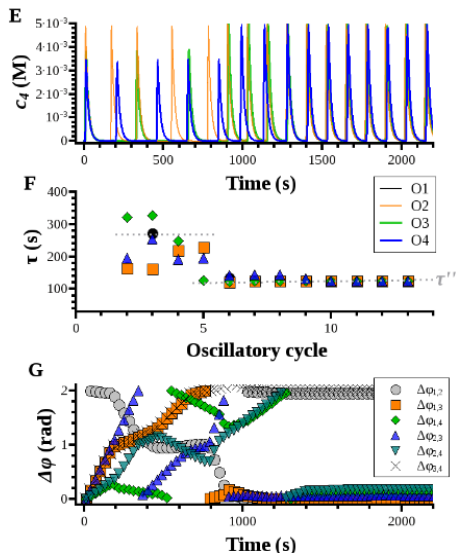

Figure 2. (A) and (E) Experimental and simulated time series for an array of $4 \mathrm{BZ}$ oscillators suspended in DEs ( $C_{4}{ }^{i}$ is the ferriin concentration of the $i$-th oscillator); (B) and (F) Experimental and simulated profiles of the oscillation period, $\tau$, as a function of the oscillatory cycle of the corresponding time series; (C) and (G) Experimental and simulated phase difference among different oscillators' couples. All the oscillating drops converge to a unique in-phase-synchronized period. In experiments, the time profile of the oscillation period decays showing a sharp period-halving transition after $\sim 500 \mathrm{~s}$ in DEs $\left(\tau^{\prime}(32 \mathrm{~s}) \rightarrow \tau^{\prime \prime}=\tau^{\prime} / 2(16 \mathrm{~s})\right)$. In simulations, increasing excitatory coupling (here $k_{x}=0.01 \mathrm{~s}^{-1}$ with $k_{W}=0.07 \mathrm{~s}^{-1}$ ) leads to an in-phase synchronization of all microoscillators after the period-halving transition compatible with experiments.(D) Schematic of the model adopted to simulate the array of oscillating w/o/w double-emulsions (DEs) (more details in $\mathrm{ESI}$ ). $\mathrm{HBrO}_{2}$ (activator), $\mathrm{Br}_{2}$ (inhibitor) are the chemical messengers able to cross the lipidic membrane of the $i$-th oscillator; $k_{x}$ and $k_{w}$ are the rate constants tuning the excitatory and inhibitory coupling, respectively. Simulations include kinetic terms that describe the removal of $\mathrm{Br}_{2}$ from the oscillatory cores due to external PVA aggregates (S), regulated by the rate constant $k_{s}$.

$\left(\Delta \varphi_{\mathrm{ij}}\right)$ progressively shifts from a random behaviour in time (scattered points in Figure $2 \mathrm{C}$ ) to a coordinated value of $\sim 2 \pi(0)$. Interestingly, the phase synchronization slightly precedes the period-halving transition as it happens in nearly identical phasecoupled oscillators where period adjustments follows the phase difference dynamics. ${ }^{20}$ This represents, at the best of our knowledge, a new synchronization scenario in the context of diffusively coupled oscillators. The main advantage of arranging the droplets in a w/o/w DEs configuration is that the relative importance of the excitatory and the inhibitory coupling can be tuned without introducing reactive species in the membranes. Here DEs are used to activate selectively an excitatory coupling, a feature that is not accessible with simple emulsions. The presence of the external aqueous shell can, in fact, favours the migration of more polar oxy-bromine species (BZ activators) among different compartments, thus increasing the excitatory contribution to the coupling mechanism. This triggers the ultimate in-phase synchronization scenario observed. In parallel, when $\mathrm{Br}_{2}$ spreads out from the oscillating core of the droplets and pass through the external water phase of the $D E$, it can associate either with PVA or sucrose. The interaction of halides with PVA and sucrose is well documented ${ }^{21}$ and, indeed, can provide a way to dump the inhibitory communication pathway without affecting the activatory species. The loss of bromine due to its sequestration in the aqueous layer is responsible for the oscillation period decrease. In previous approaches, where $\mathrm{Br}_{2}$ was prevented to migrate among the micro-oscillators, the period decay started earlier and proceeded in a smooth way without any clear synchronization (see for example the role of cholesterol in refs ${ }^{11}$ ). In contrast, DEs arrays offer a neat example of a sharp transition. The abrupt change in the oscillating frequency suggests an interplay of $\mathrm{Br}_{2}$ with a species characterized by an accelerated clock-like growth. The most probable candidate in this respect appears PVA, which is likely to form loose aggregate structures which are influenced by the co-presence of $\mathrm{Br}_{2}$ and DMPC. An indirect evidence of such a mechanism was obtained thanks to simple check experiments (see details in ESI) where the diffusive transfer of $\mathrm{Br}_{2}$ from the oil to the aqueous phases that characterizes $\mathrm{w} / \mathrm{o} / \mathrm{w}$ double emulsions was followed spectrophotometrically. The transfer dynamics showed that the presence of PVA accelerates this transfer process. In both cases (with and without PVA), we could fit the trends with a form compatible with a diffusive process $f(t) \sim \operatorname{erfc}\left[\left(x_{0}\right) /(4 D t)\right]$ (thus obtaining the concentration evolution versus the time $t$, at a given distance $x_{0}$ from the oil-water interface for a species with diffusivity $D$ ). However, to fit the curves related to experiments with PVA, we need a diffusivity of $\mathrm{Br}_{2}$ about two orders of magnitude larger than the expected value $\left(1.8 \times 10^{-5} \mathrm{~cm}^{2} \mathrm{~s}^{-1}\right)$. The enhancement of the transfer induced by the presence of PVA could be attributed to a cooperative interplay of $\mathrm{Br}_{2}$ that could seed the formation of PVA aggregates, that in turn interact with $\mathrm{Br}_{2}$ itself. To support this hypothesis, PVA water solutions were analyzed using Dynamic Light Scattering (see ESI), which revealed the presence of large, polydisperse aggregates with hydrodynamic diameter in the range from $100 \mathrm{~nm}$ up to $800-1000 \mathrm{~nm}$. After the addition of the BZ reactants the polydispersity of the whole system did not change but the aggregate size increased up to a maximum of $\sim 2700$ $\mathrm{nm}$. The interpretative picture of experimental scenario described above was tested with a minimal model where oscillating droplets, consisting of the BZ core and the surrounding double-layer shell (see Figure 2D), communicate with the closest neighbours via mass transfer ${ }^{11 a}$ of the excitatory and inhibitory species $\mathrm{HBrO}_{2}(\mathrm{X})$ and $\mathrm{Br}_{2}(\mathrm{~W})$, respectively. The oscillatory dynamics was described by using the Vanag-Epstein model ${ }^{22}$ and the relative importance of the excitatory and the inhibitory interplay among successive oscillators was tuned by the coupling constants $k_{x}$ and $k_{w}$, respectively, both depending on their membrane permeability, 
$\mathrm{P}_{\mathrm{l}}$, as $k_{l}=3 \mathrm{P}_{\mathrm{l}} / \mathrm{R}$, where $\mathrm{R}$ is the droplet radius. Reliable values for the corresponding coupling parameters typically range between $\left[10^{-4}, 10^{-3}\right] \mathrm{s}^{-1}$ for $k_{x}$ and $\left[10^{-3}, 10^{-1}\right] \mathrm{s}^{-1}$ for $k_{w} \cdot{ }^{11 \mathrm{~b},}, 15$ The clocklike growth of the concentration of the bromine sequestrating agent $S$ was modelled via a phenomenological form, $s(t)=s_{c} /\left(1+\exp \left[-t+t_{\text {ind }}\right]\right.$ ) (where $s_{c}$ is the saturation concentration of $S$ and $t_{\text {ind }}$ the induction period before the abrupt growth takes place). The bromine interacts with this species according to a general bimolecular kinetics $\mathrm{Br}_{2}+\mathrm{S} \rightarrow \mathrm{Br}_{2}-\mathrm{S}$. The equations and further details of the model are described in the ESI. We performed numerical simulations for an array of 4 coupled micro-oscillators, each with a different starting frequency determined by specific distributions of the initial concentration of the $\mathrm{BZ}$ main reactants, based on experimental values, namely $\left[\mathrm{BrO}_{3}{ }^{-}\right] \in[0.12,0.18] \mathrm{M}$ while $\left[\mathrm{H}^{+}\right]=[\mathrm{MA}]=0.3 \mathrm{M}$. The resulting kinetic model reproduces well the observed experimental dynamics as reported in Figure $2 \mathrm{E}$. The period-halving transition sharply occurs as soon as the interaction between $\mathrm{Br}_{2}$ and the related sequestration agent takes place and culminates in an inphase synchronization. The dynamics is characterized in Figure $2 \mathrm{~F}$, where the period $\mathrm{\tau}$ is reported as a function of the oscillatory cycle, and Figure 2G showing the phase difference among oscillators. The numerical exploration of the model of the oscillatory DEs indicates a drastic decrease of the oscillation period, matching a quantitative period-halving only when $k_{x}$ is increased. This supports the idea that both the inhibitor loss and an efficient excitatory coupling are needed to induce the periodhalving transition. Indeed, while simulations with large $k_{x}$ (accounting for the enhanced transfer of the activator in the water shell of DEs) are consistent with the dynamics reported here for DEs, smaller values of $k_{x}$ lead to a weaker decrement of the oscillation period and uncorrelated dynamics, as it was reported for cholesterol doped simple emulsions. ${ }^{11 a, 11 b}$ Similar frequency multiplying bifurcation was found by Vanag and Hanazaki for a single oscillator when BZ reaction was performed in a reverse microemulsion medium. ${ }^{23}$ The observed behaviour was explained in terms of $\mathrm{Br}_{2}$ migration from the water core of the emulsions to the organic continuous phase. Furthermore the period-halving bifurcation obtained in DE can be related with the transition from standing to traveling waves accompanied by a characteristic halving of the pattern wavelength, reported by Epstein and co-workers for the BZ reaction performed in reverse microemulsions. ${ }^{24}$ The two phenomena probably present the same origin and, as proposed by Epstein et al. and confirmed by our experiments, can be triggered by the collective synchronization of the spatially extended nano- or micro-oscillators, combined with the loss of bromine.

In conclusion, we have shown the paramount importance of interfaces and of selective intermediate sequestration in controlling the global behaviour in a network of diffusivelycoupled chemical oscillators. In previous works the tuning of the coupling strength was modulated by introducing different chemical species in the boundary membranes ${ }^{11 \mathrm{~b}}$ or by changing their lamellarity and thickness. ${ }^{11 a}$ Here we have presented an optimized microfluidic approach for the production of multisomes whose configuration and spatial arrangement allowed to explore new dynamical behaviours by activating an excitatory coupling not possible in single emulsions even with engineered interfaces.

\section{Conflicts of interest}

There are no conflicts to declare.

\section{Notes and references}

1. G. Ashkenasy, T. M. Hermans, S. Otto and A. F. Taylor, Chem. Soc. Rev., 2017, 46, 2543-2554.

2. a)J. J. Collins, Nature, 2003, 422, 117-118; b)N. Kopell, Science, 2003, 300, 1878-1879; c)S. H. Strogatz, Sync : the emerging science of spontaneous order, New York, NY : Hyperion, 2003.

3. a)A. F. Taylor, M. R. Tinsley, F. Wang, Z. Huang and K. Showalter, Science, 2009, 323, 614-617; b)J. A. Papin, T. Hunter, B. O. Palsson and S. Subramaniam, Nat. Rev. Mol. Cell Bio., 2005, 6, 99-111;

4. a)A. F. Taylor, Prog. React. Kinet. Mech., 2002, 27, 247-326; b)I. R. Epstein, Chem. Commun., 2014, 50, 10758-10767; c)K. Torbensen, F. Rossi, S. Ristori and A. Abou-Hassan, Lab Chip, 2017, 17, 1179-1189.

5. J. Guzowski, K. Gizynski, J. Gorecki and P. Garstecki, Lab Chip, 2016, 16, 764 772

6. T. Bánsági, V. K. Vanag and I. R. Epstein, Science, 2011, 331, 1309-1312.

7. R. Tomasi, J.-M. Noel, A. Zenati, S. Ristori, F. Rossi, V. Cabuil, F. Kanoufi and A Abou-Hassan, Chem. Sci., 2014, 5, 1854-1859.

8. a)Y. Hu and J. Pérez-Mercader, Coll. Surf. B: Biointerf., 2016, 146, 406-414; b)Q. Shao, S. Zhang, Z. Hu and Y. Zhou, Angew. Chem. Int. Ed., doi:10.1002/anie.202007840

9. a)M. Toiya, V. K. Vanag and I. R. Epstein, Angew. Chem. Int. Ed., 2008, 47, 7753-7755; b)M. Toiya, H. O. González-Ochoa, V. K. Vanag, S. Fraden and I. R. Epstein, J. Phys. Chem. Lett., 2010, 1, 1241-1246.

10. a)T. Litschel, M. M. Norton, V. Tserunyan and S. Fraden, Lab Chip, 2018, 18, 714-722; b)S. Thutupalli and S. Herminghaus, Eur. Phys. J. E, 2013, 36, 91; c)S. Thutupalli, S. Herminghaus and R. Seemann, Soft Mat., 2011, 7, 1312-1320.

11. a)M. A. Budroni, K. Torbensen, S. Ristori, A. Abou-Hassan and F. Rossi, J. Phys. Chem. Lett., 2020, 11, 2014-2020; b)K. Torbensen, S. Ristori, F. Rossi and A. Abou-Hassan, J. Phys. Chem. C., 2017, 121, 13256-13264; c)K. Torbensen, F. Rossi, O. L. Pantani, S. Ristori and A. Abou-Hassan, The J. Phys. Chem. B, 2015, 119, 10224-10230.

12. E. Di Cola, K. Torbensen, I. Clemente, F. Rossi, S. Ristori and A. Abou-Hassan, Langmuir, 2017, 33, 9100-9105.

13. a)T. Narayanan, M. Sztucki, P. Van Vaerenbergh, J. Leonardon, J. Gorini, L. Claustre, F. Sever, J. Morse and P. Boesecke, J. Appl. Crystallogr., 2018, 51, 1511-1524; b)I. Clemente, K. Torbensen, E. Di Cola, F. Rossi, S. Ristori and A. Abou-Hassan, RSC Adv., 2019, 9, 33429-33435.

14. a)N. Li, J. Delgado, H. O. González-Ochoa, I. R. Epstein and S. Fraden, Phys. Chem. Chem. Phsy., 2014, 16, 10965-10978; b)D. A. Safonov and V. K. Vanag, Phys. Chem. Chem. Phsy., 2018, 20, 11888-11898.

15. F. Rossi, A. Zenati, S. Ristori, J.-M. Noîl, V. Cabuil, F. Kanoufi and A. AbouHassan, Int. J. Unconv. Comput., 2015, 11, 23-36.

16. a)D. Needham, Nat. Nanotech., 2011, 6, 761-762; b)T. Trantidou, M. Friddin, Y. Elani, N. J. Brooks, R. V. Law, J. M. Seddon and O. Ces, ACS Nano, 2017, 11, 6549-6565

17. a)H. C. Shum, A. Bandyopadhyay, S. Bose and D. A. Weitz, Chem. Mater., 2009, 21, 5548-5555; b)S. Ding, C. A. Serra, T. F. Vandamme, W. Yu and N. Anton, Control Release, 2019, 295, 31-49.

18. K. Torbensen and A. Abou-Hassan, J. Flow. Chem., 2015, 5, 234-240.

19. M. A. Budroni and F. Rossi, J. Phys. Chem. C., 2015, 119, 9411-9417.

20. a)A. T. Winfree, J Theor Biol, 1967, 16, 15-42; b)Y. Kuramoto, Chemical Oscillations, Waves, and Turbulence, Springer, Berlin, Heidelberg, 1984.

21. a)D. K. Gaskins, E. E. Pruc, I. R. Epstein and M. Dolnik, Phys. Rev. Lett., 2016, 117, 056001; b) P. Stano, F. Wodlei, P. Carrara, S. Ristori, N. Marchettini and F. Rossi, in Advances in Artificial Life and Evolutionary Computation, eds. C. Pizzuti and G. Spezzano, Springer International Publishing, Cham, 2014, pp. 58-74

22. V. K. Vanag and I. R. Epstein, J. Chem. Phys., 2009, 131, 104512.

23. V. K. Vanag and I. Hanazaki, J. Phys. Chem., 1995, 99, 6944-6950.

24. A. Kaminaga, V. K. Vanag and I. R. Epstein, Phys. Rev. Lett., 2005, 95, 058302. 